\title{
Smoking Cause Specific Lung Cancer-Evidence from Non-Smoking Lung Adenocarcinoma
}

\author{
Ning Li, Bin Qu, Kang Shao, Zhaoli Chen, Fengwei Tan, Xiaogang Tan, Baozhong Li, Jiwen Wang, \\ Jie $\mathrm{He}^{*}$
}

Department of Thoracic Surgery, Cancer Hospital and Institute, Chinese Academy of Medical Sciences, Beijing, China. Email: *Dr.hejie@gmail.com

Received July $13^{\text {th }}$, 2012; revised August $25^{\text {th }}, 2012$; accepted September $16^{\text {th }}, 2012$

\begin{abstract}
Introduction: Smoking and non-smoking lung cancer have many differences in clinical feature. But those may be the result of interference due to differences in pathological type, as most smoking patients suffer squmous cell lung cancer and non-smokings tend to get adenocarcinoma. This study was conducted on the specific histological type-lung adenocarcinoma - to avoid histological bias and to reveal the true effect of smoking. Methods: A total of 2222 patients with lung adenocarcinoma confirmed by histological or cytological evidence were enrolled from January 1, 1999 to December 31, 2004. Differences in clinical features and prognosis between non-smoking and smoking patients were analyzed. Chi-square test was used for univariate comparisons. Univariate probability of survival was computed using Kaplan-Meier estimate and compared to using the log-rank test. Cox proportional hazards regression analysis was used to evaluate the risk of death. Results: There were 777 current smokers (34.96\%), 197 former smokers (8.87\%) and 1248 non-smoking patients (56.17\%). 860 non-smoking patients (68.91\%) were female, compared with $6.31 \%$ among current smokers and $4.06 \%$ among former smokers $(\mathrm{p}<0.001)$. Non-smoking patients had an earlier age at diagnosis (p $<0.001)$ and a better response to chemotherapy $(\mathrm{p}<0.001)$ compared to current smoking patients. Current smoking correlated with lower cell differentiation $(p<0.001)$ and worse prognosis $(p=0.0024)$. After multivariate analysis, smoking was identified as an independent negative prognostic factor (HR, 1.302; 95\% CI, $1.011-1.6780, \mathrm{p}=0.041$ ). No difference in prognosis was observed according to smoking conditions in smoking patients. Conclusions: Significent differences exist in clinical features and prognosis between non-smoking and smoking lung adenocarcinoma patients. There is a strong evidence that non-smoking lung adenocarcinoma should be regard as different disease.
\end{abstract}

Keywords: Lung Adenocarcinoma; Cigarette Smoke; Differentiation; Chemotherapy; Prognosis

\section{Introduction}

Lung cancer is the most lethal cancer in the world with over 1 million deaths each year [1]. Cigarette smoking was regarded as the most important carcinogenic factor of lung cancer, which has been well established from epidemiological evidence [2]. However, over 50 percent of female and 15 percent of male lung cancer patients never smoke cigarette [3]. The incidence of lung cancer in non-smoker was even higher than several high incidence cancers, such as: cervix, pancreas and prostate cancer worldwide [3].

Smoking can not only increase the lung cancer incidence but also affect the lung cancer patient's clinical feature and prognosis. Smoking lung cancer patients have many differences in gender distribution, clinical features, pathology result and molecular mechanism comparing to non-smoking lung cancer $[4,5]$. Smoking lung cancer

${ }^{*}$ Corresponding author. patients tend to be diagnosed at older age, present with early stage, usually be afflicted with squamous cell lung cancer, and have a better prognosis [6-8]. Non-smoking lung cancer have a higher rate of mutation in Epidermal growth factor receptor (EGFR) and a better response to the EGFR Tyrosine Kinase Inhibitor: Gifitinib and Erlotinib $[9,10]$, while have less mutations in P53 compared with smoking lung cancer $[4,11]$. Therefore, many scholarssuggested that lung cancer in never smokers is a "different" disease, with a different aetiology and a different natural history [5,6].

A small defect exists in the evidences of this hypothesis. Previous researches about clinical features and survival analysis mostly focus on all categories of lung cancer [7] or non-small cell lung cancer (NSCLC) $[6,8,12]$. Over 70 percent smoking patients suffer from squamous cell lung cancer and most non-smoking patients suffer from lung adenocarcinoma [13]. Many distinctions exist in clinical features between subtypes of lung cancer, and 
histology, such as squamous cell or adenocarcinoma have been recognized as a predictive factor for response to chemotherapy and prognosis [14]. So, differences between smoking and non-smoking lung cancer might be confounded by the differences between squamous cell cancer and adenocarcinoma.

To avoid any bias, we performed a retrospective study of lung adenocarcinoma, which contains the most part of non-smoking patients. We compared all detectable variance in clinical features and prognosis between nonsmoking and smoking patients, to investigate the true effect of smoking to the lung adenocarcinoma.

\section{Patients and Methods}

Current respective study was conducted in the Cancer hospital, Chinese Academy of Medical Sciences (CAMS). Patients had primary lung adenocarcinoma diagnosed between 1 January 1999 and 31 December 2004.

All patients diagnosed consecutively with primary lung adenocarcinoma, which was confirmed by histological or cytological examination. To avoid the influence from different histological subtype, adenosquamous carcinoma and bronchiolo-alveolar carcinoma were excluded. To avoid the influence form different ethnic groups, only Chinese patients were enrolled. Patients were excluded if smoking history was not available in case record. Individual case records were reviewed. In addition to smoke history, age, gender, lung cancer family history, American Joint Committee on Cancer (AJCC) stage at diagnosis and type of treatment were recorded. When surgery was performed as initial treatment, the metastasis of lymph nodes ( $\mathrm{N}$ stage), differentiation of adenocarcinoma cells were collected as well. When chemotherapy was performed as initial treatment in advanced patients (Stage IIIB and IV), the clinical response after four cycles to first-line chemotherapy were also recorded as four levels: Complete Response (CR), Partial Response (PR), Stable Disease (SD) and Progress Disease (PD), according to RECIST critical. Survival data were obtained from follow-up group in thoracic department cancer hospital CAMS. All patients were notified and understand that their clinical information might be used for clinical research and analysis, before they got the therapy. And all included in this study signed the authorizations form. The study was approved by the medical ethics committee of Cancer Institute and Hospital, CAMS.

Smoking history contains smoking conditions, such as: how many years of smoking, how many cigarettes smoked per day, and how many years since quitting smoke. Patients who were smokers during diagnosis or stop smoking less than 1 year were collected in "current smoker" group [5]. Patients who quit smoking more than one year were classified as "former smoker". Patients who have a lifetime exposure of less than 100 cigarettes or never smoked in the past were termed as "never smoker".

According to smoking habits, chi-square test was used for univariate comparisons of patients, disease and treatment-related characteristics. Cox proportional hazards regression analysis was used to evaluate the risk of death between current, former and never smoking patients, while adjusting for other prognostic factors including age at diagnosis, gender, lung cancer family history, disease stage at diagnosis and initial treatment. Univariate probability of survival was computed using Kaplan-Meier estimate and compared using the log-rank test. P-Value of 0.05 was considered significant for all analysis.

\section{Results}

A total of 2222 patients were enrolled and met study selection criteria. 777 patients (34.96\%) were current smokers, 1248 patients (56.17\%) were never smokers, and 197 patients (8.87\%) who quit smoking for at least one year were termed as "former smokers". Demographic and clinical features of each group were listed in Table 1.

The overwhelming majority of current smokers and former smokers were males, $93.69 \%$ in current smokers and $95.94 \%$ in former smokers. On the other hand, only $31.09 \%$ patients in never smoker group were males. Non-smoking patients were diagnosed at median age of 56 years old, which was younger than current and former smokers $(\mathrm{p}<0.001)$. Former smokers tend to present with earlier disease than current and never smokers $(\mathrm{p}=$ 0.016). Operations were preformed in 503 current smokers (64.74\%), 153 former smokers (77.66\%) and 794 never smokers (63.62\%). Differentiation in adenocarcinoma cells was evaluated in the tissue samples resects in operations. More proportion of poor differentiation was found in current smoking patients (56.39\%) than former (47.37\%) and never $(35.87 \%)$ smokers $(\mathrm{p}<0.001)$. There was a weak significant difference in N2 lymph nodes metastasis $(p=0.146)$ between three groups. Complete response and partial response were seen in 165 (41.88\%) non-smoking patients, but only in $41(23.56 \%)$ current and $8(26.67 \%)$ former smoking patients $(\mathrm{p}<0.001)$.

Overall survival is shown in Figure 1. The 3-year overall survival rates for current smoker, former smoker and never smoker were $23.63 \%, 28.15 \%$ and $33.55 \%$. The 5-year overall survival rate were $19.85 \%, 23.06 \%$ and $22.93 \%$, respectively $(\mathrm{p}=0.0024)$. Interestingly, former smokers had similar survival as never smokers, and much better than current smokers in patients with early stage (stage I and II) (Figure 2).

As smoking patients had different smoking conditions, additional analysis were performed out within the current smoking group to examine the impact of smoking conditions on prognosis of smoking patients. There were no 
Table 1. The clinical features of lung adenocarcinoma patients.

\begin{tabular}{|c|c|c|c|c|c|c|c|}
\hline \multirow{2}{*}{ Variable } & \multicolumn{2}{|c|}{ Current Smokers $(n=777)$} & \multicolumn{2}{|c|}{ Former Smokers $(n=197)$} & \multicolumn{2}{|c|}{ Never Smokers $(n=1248)$} & \multirow{2}{*}{$p$ value } \\
\hline & No. & $\%$ & No. & $\%$ & No. & $\%$ & \\
\hline \multicolumn{8}{|l|}{ Gander } \\
\hline Male & 728 & $93.69 \%$ & 189 & $95.94 \%$ & 388 & $31.09 \%$ & \multirow[t]{2}{*}{$\mathrm{p}<0.001$} \\
\hline Female & 49 & $6.31 \%$ & 8 & $4.06 \%$ & 860 & $68.91 \%$ & \\
\hline \multicolumn{8}{|l|}{ Age at diagnosis } \\
\hline Median & \multicolumn{2}{|c|}{59} & \multicolumn{2}{|c|}{66} & \multicolumn{2}{|c|}{56} & $\mathrm{p}<0.001$ \\
\hline Range & \multicolumn{2}{|c|}{$23-86$} & \multicolumn{2}{|c|}{$40-83$} & \multicolumn{2}{|c|}{$20-84$} & \\
\hline \multicolumn{8}{|c|}{ AJCC stage at diagnosis $(\mathrm{n}=2144)$} \\
\hline I & 200 & $26.74 \%$ & 57 & $31.32 \%$ & 278 & $22.90 \%$ & \multirow{4}{*}{$\mathrm{p}=0.016$} \\
\hline II & 64 & $8.56 \%$ & 17 & $9.34 \%$ & 108 & $8.90 \%$ & \\
\hline III & 176 & $23.53 \%$ & 54 & $29.67 \%$ & 342 & $28.17 \%$ & \\
\hline IV & 308 & $41.18 \%$ & 54 & $29.67 \%$ & 486 & $40.03 \%$ & \\
\hline \multicolumn{8}{|l|}{ Treatment } \\
\hline Operation & 503 & $64.74 \%$ & 153 & $77.66 \%$ & 794 & $63.62 \%$ & \multirow[t]{2}{*}{$\mathrm{p}=0.001$} \\
\hline Chemotherapy/Others & 274 & $35.26 \%$ & 44 & $22.34 \%$ & 454 & $36.38 \%$ & \\
\hline \multicolumn{8}{|c|}{ Differentiation of adenocarcinoma cells $(n=1323$ ) } \\
\hline Low differentiation & 256 & $56.39 \%$ & 63 & $47.37 \%$ & 264 & $35.87 \%$ & \multirow{3}{*}{$\mathrm{p}<0.001$} \\
\hline Middle differentiation & 166 & $36.56 \%$ & 57 & $42.86 \%$ & 404 & $54.89 \%$ & \\
\hline High differentiation & 32 & $7.05 \%$ & 13 & $9.77 \%$ & 68 & $9.24 \%$ & \\
\hline \multicolumn{8}{|c|}{ Metastasis of lymph nodes $(n=1298)$} \\
\hline No & 205 & $46.49 \%$ & 59 & $45.74 \%$ & 288 & $39.56 \%$ & \multirow{3}{*}{$\mathrm{p}=0.146$} \\
\hline N1 & 69 & $15.65 \%$ & 17 & $13.18 \%$ & 117 & $16.07 \%$ & \\
\hline N2 & 167 & $37.87 \%$ & 53 & $41.09 \%$ & 323 & $44.37 \%$ & \\
\hline
\end{tabular}

significant difference in prognosis of smokers according to years of smoking, number of cigarettes smoked per day and the smoking index (Figure 3).

Table 2 summarized the hazard ratios and significance of the impact of clinical features. The risk of dying for current smokers remained higher compared with neversmokers after adjusting for gender, clinical stage, treatment and family history (HR: 1.302; 95\% CI: 1.011 1.678; $\mathrm{p}=0.041$ ). Current smoking status was an independent variable for poor prognosis in lung adenocarcimona.

\section{Discussion}

Non-smoking lung related cancer has many differences compared with smoking related lung cancer, from etiological factors to biological behavior. Non-smoking lung cancer might result from other risk factors, such as: preexisting lung disease, endocrine factors, family history, radiation, air pollution and environmental tobacco smoking [13]. Cancers induced by different carcinogenic agents might be variables in biological behavior. With different ability of proliferation, differentiation, migra- 
Table 2. Cox proportional hazards regression analysis of factors associated with survival in patients with lung adenocarcinoma.

\begin{tabular}{|c|c|c|c|c|}
\hline Variable & Categories/Unite & Hazard Ratio & $95 \%$ CI & $p$ value \\
\hline & Never smoker & 1 & & \\
\hline \multirow[t]{2}{*}{ Cigarette smoke } & Former smoker & 1.289 & $0.894-1.858$ & 0.175 \\
\hline & Current smoker & 1.302 & $1.011-1.678$ & 0.041 \\
\hline \multirow{2}{*}{ Gender } & Female & 1 & & \\
\hline & Male & 1.129 & $0.875-1.457$ & 0.352 \\
\hline \multirow{2}{*}{ Family history } & Negative & 1 & & \\
\hline & Positive & 0.635 & $0.699-1.244$ & 0.635 \\
\hline \multirow{3}{*}{ Treatment } & Operation & 1 & & \\
\hline & Others & 1.410 & $0.925-2.151$ & 0.110 \\
\hline & Stage I & 0.132 & $0.081-0.215$ & $<0.001$ \\
\hline \multirow{3}{*}{ TNM stage } & Stage II & 0.419 & $0.246-0.713$ & 0.001 \\
\hline & Stage III & 0.752 & $0.490-1.154$ & 0.192 \\
\hline & Stage IV & 1 & & \\
\hline
\end{tabular}

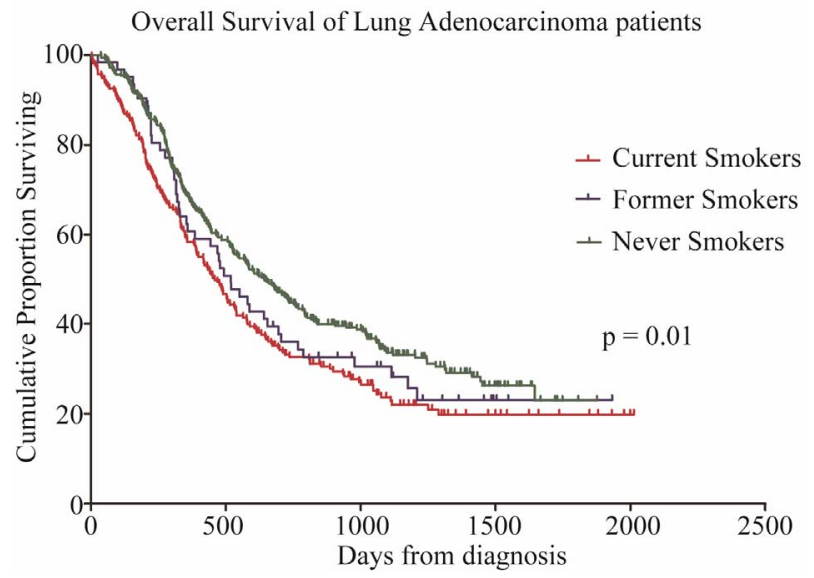

Figure 1. Kaplan-Meier survival curves stratified by smoking status in patients with lung adenocarcinoma.

tion and invasion, even though cancer cells had similar appearance, they could be regarded as "different cancer". It is a feasible method to investigate the biological features of non-smoking related lung cancer, to analyze the clinical features of patients, and then compare with smoking patients. Differences in age, gender, initial stage, response to chemotherapy and prognosis between nonsmoking and smoking patients had been found in previous studies [5,6].

However, several influencing factors should not be ignored. Distinctions in pathology have been established by previous studies. Non-smoking lung cancer patients tend to suffer from adenocarcinoma. Patients with lung adenocarcinoma have many differences in gender, age at diagnosis, response to chemotherapy and prognosis compared to those with squamous cell lung cancer [14]. The ethnic difference is another important factor. Asian female had much lower smoking rate and higher lung cancer incidence compared to American and European female, and they are more sensitive to the EGFR Tyrosine Kinase Inhibitor [5]. Japanese ethnicity is independent favorable prognostic factors when compared with Caucasian ethnicity in NSCLC [15]. Another factor is the choice of the treatment: each institution or oncologist chose method mainly based on local policies, cost or convenience of the therapeutic schedule, which could affect the prognosis of the patients. Therefore, we conducted current study in a specific histological pathology, in a specific ethnic group and in a specific institute, to avoid those influences.

Non-smoking patients were diagnosed earlier at median age of 56 years old compared with current and former smoking patients. Former studies from Asia countries had similar results [6], while studies from United States and Europe did not [5]. Those facts indicate that non-smoking lung cancer in Asia might not be resulted from environment tobacco smoking, as patients do not have enough exposure to tobacco smoking [16]. Differences between those two cohorts, such as genetic factors 

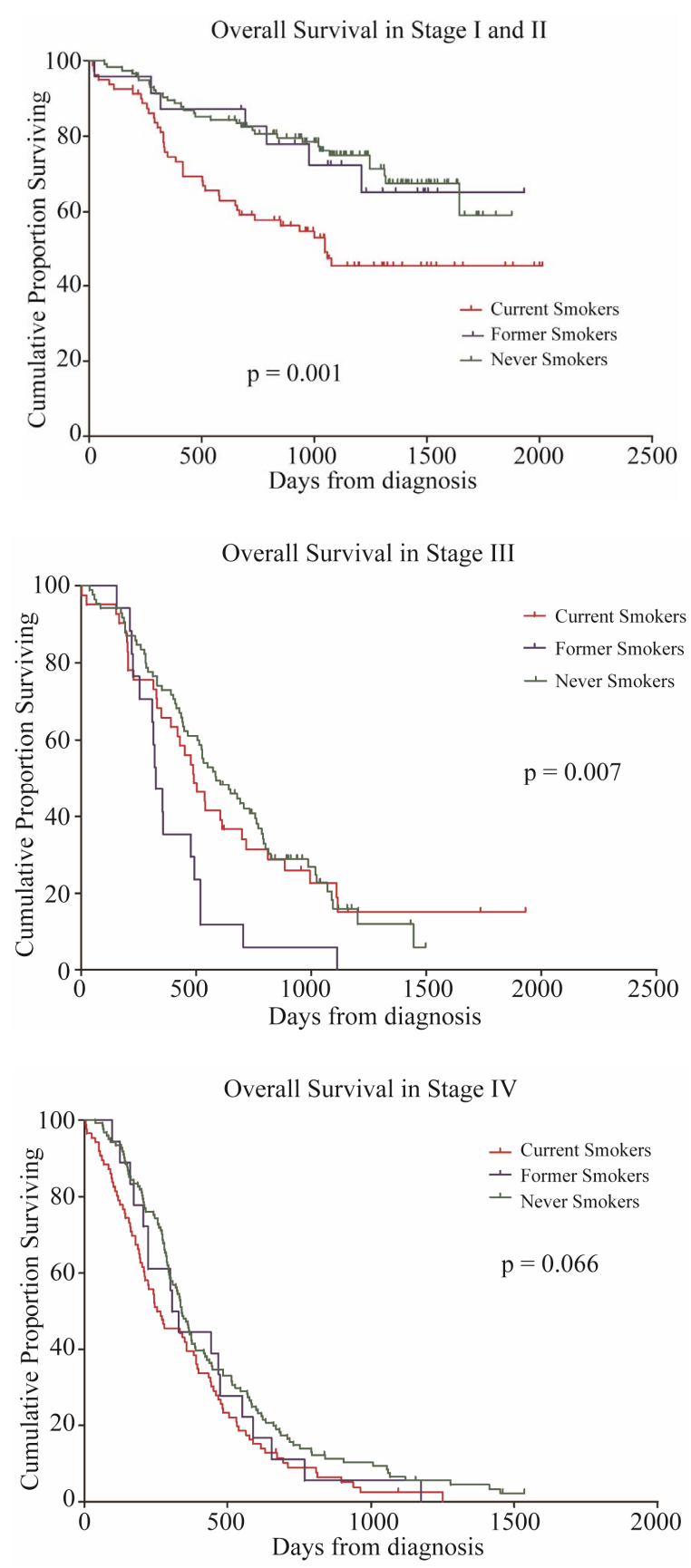

Figure 2. Kaplan-Meier survival curves stratified by smoking status in patients with Stages I and II, III, and IV.

and environment factors are important in lung cancer carcinogenesis. Most patients in never smoking group are female. Similar results were obtained in many previous studies [6], which indicated the female is more sensitive to carcinogen among non-smokers. Direct evidence comes from incidence investigation: incidence of female patients with non-smoking lung cancer is 14.4 - 20.8/100000, while 4.8 - 13.7/100000 in male $[17,18]$.

Here we reported that histological differentiation of cancer cells is different among patients with diverse
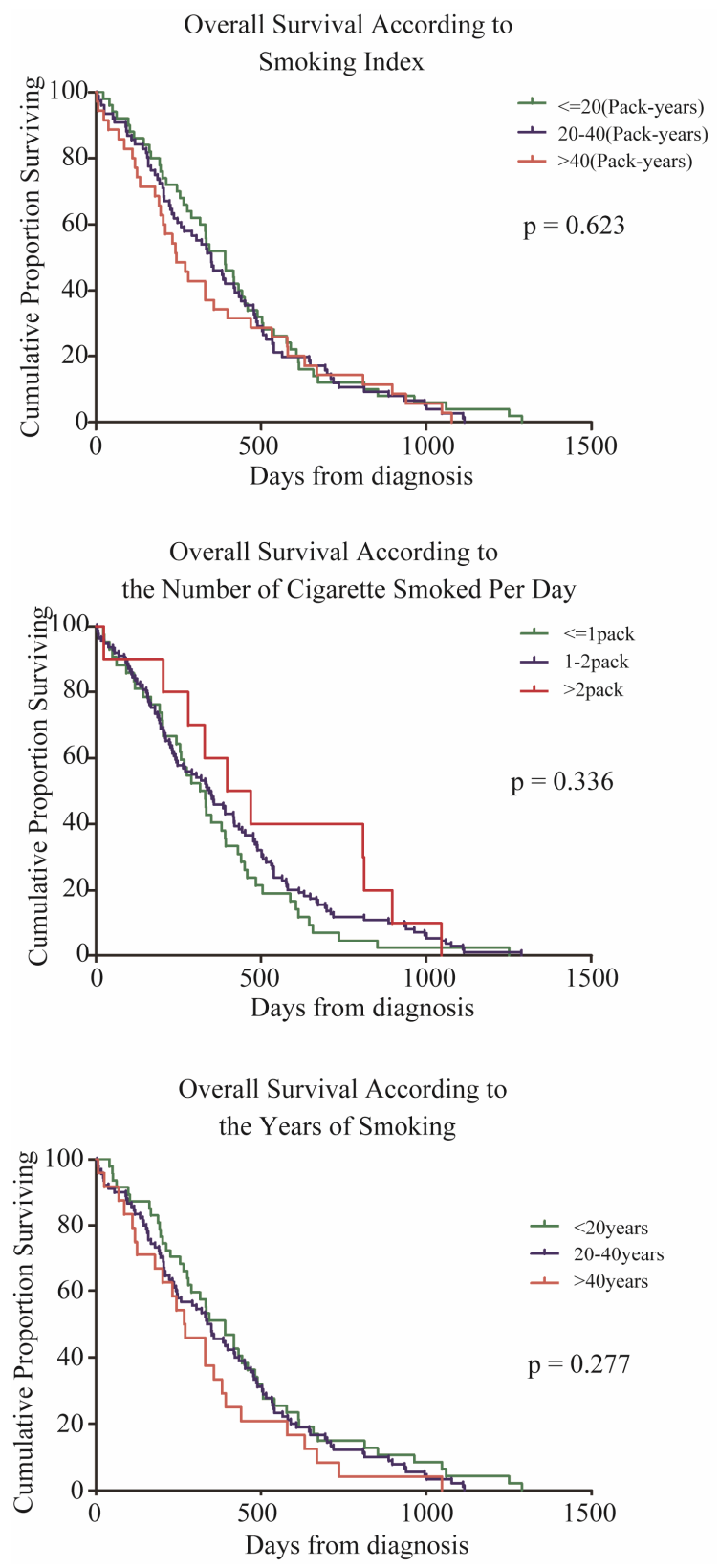

Figure 3. Kaplan-Meier survival curves stratified by smoking conditions in smoking patients.

smoking conditions. Non-smoking patients usually had a better differentiation than smokers, which was a direct pathomorphological evidence for "different cancer". The smoke of cigarette contains more than 4500 chemical compounds. Many of them affect the cell differentiation in vitro and in vivo [19], which could explain poorer differentiation in smoking patients.

We demonstrated patients in never smoker group had better response to front line chemotherapy than those in the other two groups. Tsao et al. had similar results. They analyzed 873 advanced non small cell lung cancer patients who received chemotherapy, and found never- 
smokers had higher response rates and lower rates of progressive disease than former and current smokers [8]. As chemotherapy drugs work directly on the cancer cells, different responses indicate different intracellular mechanisms [20]. Previous study shows, compared with people who never smoke, people with lung adenocarcinoma who smoked or stopped smoking less than 25 years ago have significant less EGFR mutations in exons 19 and 21 [21]. A study of comparing gene expression profiles in never smokers and smokers with lung adenocarcinoma by Powell et al. reported that there might be different pathways of cell transformation and tumor formation in two groups, with many differences in gene expression. [22] Those are strong evidences for the "different cancers".

Surviving time of patients is considered as the most important characteristics in cancer research. Different subtypes of cancer usually have dissimilar prognosis, such as: lung squamous cell cancer and adenocarcinoma; gastric signet-ring cell carcinoma and adenocarcinoma; hepatic cellular cancer and hepatobiliary cancer. Nonsmoking lung adenocarcinoma patients had a better prognosis than current and former smokers. Interestingly, former smoking patients had similar survival with nonsmoking patients and much better than current smoking patients in the early stage, which is a good message for patients who quit smoking. Study from Nordquist et al. reported 5 years survival were $16 \%$ for current smokers and $23 \%$ for never-smokers in 654 patients with lung adenocarcinoma [23]. Similar results were reported by Tammemagi et al., they studied 1155 lung cancer patients and found cigarette smoking was an important independent predictor of shortened lung cancer survival [7]. Bryant et al. also found never smoker patients with earlystage cancer have a significantly better survival rate than smokers [24]. As expected, in current study, we found smoking conditions such as the number of cigarettes smoked per day, years of smoking and the smoking index do not affect the prognosis. The comorbidity of smoking such as chronic obstructive pulmonary disease, hypertension, atherosclerosis and aortic aneurysm are more serious in heavy smoker; which may indicate that the difference in prognosis was not because of the comorbidities but the nature of cancer. We can infer that lung cancer caused by smoking is the same type of cancer, which has no correlation in prognosis with cigarette smoke burden. With difference in prognosis, non-smoking lung adenocarcinoma could be considered as a different cancer.

In summary, we demonstrate significant differences between non-smoking and smoking patients with lung adenocarcinoma in age at diagnosis, gender, initial stage at diagnosis, cell differentiation, response to chemotherapy and prognosis. Furthermore, we suggest non-smoking and smoking lung adenocarcinoma could be considered as different cancers with many distinctions in clini- cal features.

\section{REFERENCES}

[1] A. Jemal, T. Murray, E. Ward, et al., "Cancer Statistics, 2005,” CA-A Cancer Journal for Clinicians, Vol. 55, No. 1, 2005, pp. 10-30. doi:10.3322/canjclin.55.1.10

[2] E. L. Wynder and E. A. Graham, "Landmark Article May 27, 1950: Tobacco Smoking as a Possible Etiologic Factor in Bronchiogenic Carcinoma. A Study of Six Hundred and Eighty-Four Proved Cases,” JAMA, Vol. 253, No. 20, 1985, pp. 2986-2994.

doi:10.1001/jama.1985.03350440064033

[3] D. M. Parkin, F. Bray, J. Ferlay and P. Pisani, "Global Cancer Statistics, 2002," CA—A Cancer Journal for Clinicians, Vol. 55, No. 2, 2005, pp. 74-108.

doi:10.3322/canjclin.55.2.74

[4] R. Gealy, L. Zhang, J. M. Siegfried, J. D. Luketich and P. Keohavong, "Comparison of Mutations in the p53 and K-Ras Genes in Lung Carcinomas from Smoking and Nonsmoking Women,” Cancer Epidemiology, Biomarkers \& Prevention, Vol. 8, No. 4, 1999, pp. 297-302.

[5] S. Sun, J. H. Schiller and A. F. Gazdar, "Lung Cancer in Never Smokers-A Different Disease,” Nature Reviews Cancer, Vol. 7, 2007, pp. 778-790. doi:10.1038/nrc2190

[6] C. K. Toh, F. Gao, W. T. Lim, et al., "Never-Smokers with Lung Cancer: Epidemiologic Evidence of a Distinct Disease Entity,” Journal of Clinical Oncology, Vol. 24, No. 34, 2006, pp. 2245-2251.

doi:10.1200/JCO.2005.04.8033

[7] C. M. Tammemagi, C. Neslund-Dudas, M. Simoff and P. Kvale, "Smoking and Lung Cancer Survival: The Role of Comorbidity and Treatment," Chest, Vol. 125, No. 1, 2004, pp. 27-37. doi:10.1378/chest.125.1.27

[8] A. S. Tsao, D. Liu, J. J. Lee, M. Spitz and W. K. Hong, "Smoking Affects Treatment Outcome in Patients with Advanced Nonsmall Cell Lung Cancer," Cancer, Vol. 106, No. 11, 2006, pp. 2428-2436.

doi:10.1002/cncr.21884

[9] T. J. Lynch, D. W. Bell, R. Sordella, et al., “Activating Mutations in the Epidermal Growth Factor Receptor Underlying Responsiveness of Non-Small-Cell Lung Cancer to Gefitinib,” New England Journal of Medicine, Vol. 350, No. 21, 2004, pp. 2129-2139. doi:10.1056/NEJMoa040938

[10] S. W. Han, T. Y. Kim, P. G. Hwang, et al., "Predictive and Prognostic Impact of Epidermal Growth Factor Receptor Mutation in Non-Small-Cell Lung Cancer Patients Treated with Gefitinib,” Journal of Clinical Oncology, Vol. 23, No. 16, 2005, pp. 2493-2501. doi:10.1200/JCO.2005.01.388

[11] S. N. Rodin and A. S. Rodin, "On the Excess of G --> T Transversions in the p53 Gene in Lung Cancer Cell Lines. Reply to Pfeifer and Hainaut," Mutation Research, Vol. 545, No. 1-2, 2004, pp. 141-147. doi:10.1016/j.mrfmmm.2003.09.008

[12] C. K. Toh, E. H. Wong, W. T. Lim, et al., "The Impact of Smoking Status on the Behavior and Survival Outcome of 
Patients with Advanced Non-Small Cell Lung Cancer: A Retrospective Analysis,” Chest, Vol. 126, No. 6, 2004, pp. 1750-1756. doi:10.1378/chest.126.6.1750

[13] J. Subramanian and R. Govindan, "Lung Cancer in Never Smokers: A Review,” Journal of Clinical Oncology, Vol. 25, No. 5, 2007, pp. 561-570. doi:10.1200/JCO.2006.06.8015

[14] G. Selvaggi and G. V. Scagliotti, "Histologic Subtype in NSCLC: Does It Matter?” Oncology, Vol. 23, No. 13, 2009, pp. 1133-1140.

[15] T. Kawaguchi, A. Matsumura, S. Fukai, et al., "Japanese Ethnicity Compared with Caucasian Ethnicity and NeverSmoking Status Are Independent Favorable Prognostic Factors for Overall Survival in Non-small Cell Lung Cancer: A Collaborative Epidemiologic Study of the National Hospital Organization Study Group for Lung Cancer (NHSGLC) in Japan and a Southern California Regional Cancer Registry Databases,” Journal of Thoracic Oncology, Vol. 5, No. 7, 2010, pp. 1001-1010.

[16] L. A. Loeb, V. L. Ernster, K. E. Warner, J. Abbotts and J. Laszlo, "Smoking and Lung Cancer: An Overview," Cancer Research, Vol. 44, No. 12, 1984, pp. 5940-5958.

[17] H. A. Wakelee, E. T. Chang, S. L. Gomez, et al., "Lung Cancer Incidence in Never Smokers,” Journal of Clinical Oncology, Vol. 25, No. 5, 2007, pp. 472-478. doi:10.1200/JCO.2006.07.2983

[18] L. C. Koo and J. H. Ho, "Worldwide Epidemiological Patterns of Lung Cancer in Nonsmokers," International Journal of Epidemiology, Vol. 19, Suppl. 1, 1990, pp. S14-S23.
[19] L. S. Wistuba II, C. Behrens, et al., "Molecular Damage in the Bronchial Epithelium of Current and Former Smokers," Journal of the National Cancer Institute, Vol. 89, No. 18, 1997, pp. 1366-1373. doi:10.1093/jnci/89.18.1366

[20] L. Mao, J. S. Lee, J. M. Kurie, et al., "Clonal Genetic Alterations in the Lungs of Current and Former Smokers,” Journal of the National Cancer Institute, Vol. 89, No. 12, 1997, pp. 857-862. doi:10.1093/jnci/89.12.857

[21] D. Pham, M. G. Kris, G. J. Riely, et al., "Use of Cigarette-Smoking History to Estimate the Likelihood of Mutations in Epidermal Growth Factor Receptor Gene Exons 19 and 21 in Lung Adenocarcinomas,” Journal of Clinical Oncology, Vol. 24, 2006, pp. 1700-1704. doi:10.1200/JCO.2005.04.3224

[22] C. A. Powell, A. Spira, A. Derti, et al., "Gene Expression in Lung Adenocarcinomas of Smokers and Nonsmokers," American Journal of Respiratory Cell and Molecular Biology, Vol. 29, No. 2, 2003, pp. 157-162. doi:10.1165/rcmb.2002-0183RC

[23] L. T. Nordquist, G. R. Simon, A. Cantor, W. M. Alberts and G. Bepler, "Improved Survival in Never-Smokers vs Current Smokers with Primary Adenocarcinoma of the Lung,” Chest, Vol. 126, No. 2, 2004, pp. 347-351. doi:10.1378/chest.126.2.347

[24] A. Bryant and R. J. Cerfolio, "Differences in Epidemiology, Histology, and Survival between Cigarette Smokers and Never-Smokers Who Develop Non-Small Cell Lung Cancer,” Chest, Vol. 132, No. 1, 2007, pp. 185-192. doi:10.1378/chest.07-0442 